Irish Math. Soc. Bulletin

Number 72, Winter 2013, 29-30

ISSN 0791-5578

\title{
SPACES OF POLYNOMIALS OF DEGREE AT MOST $n$ AND THEIR GEOMETRY
}

\author{
ANTHONY BROWN
}

This is an abstract of the $\mathrm{PhD}$ thesis Spaces of Polynomials of Degree at most $n$ and their Geometry written by Anthony Brown under the supervision of Dr. Christopher Boyd at the School of Mathematical Sciences, University College Dublin and submitted in September 2011.

In this thesis we examine the geometry of spaces of polynomials of degree at most $n$. In many cases our results are stronger than the corresponding result in the homogeneous case, in some they are similar, while others are weaker.

We start by finding appropriate definitions for our spaces of integral and nuclear polynomials. This enables us to obtain duality relations between both these spaces and the space of all approximable polynomials and the space of all continuous polynomials of degree $n$. By defining particular norms on direct sums of tensors products we are also able to obtain isometric preduals of our space of integral polynomials and the space of all continuous polynomials of degree $n$.

Many of the results which follow depend on whether the underlying field of scalars is real or complex. In the the real case we obtain Smul'yan type theorems, where we give conditions which are equivalent to either the Fréchet or Gâteaux differentiability of the norm of the predual of our space of integral polynomials. These results lead us naturally to a complete characterisation of the extreme points of the unit ball of our space of integral polynomials. We show that the characterisation in the non-homogeneous case is different to that observed in the homogeneous case. This characterisation is then used to prove several Banach-Stone type theorems which express

2010 Mathematics Subject Classification. 46G25, 46A20, 46E15, 46M05.

Key words and phrases. Polynomials between Banach spaces, Duality theory.

Received on 13-12-2013.

Support from University College Dublin through an Ad Astra Research Scholarship is gratefully acknowledged. 
isometric isomorphisms between spaces of polynomials in terms of isometric isomorphisms between the duals of the spaces which are the domains of the polynomials. By showing that the centralizer of the predual of our space of integral polynomials is trivial we are able to extend these theorems to characterise isometric isomorphisms between spaces of vector valued polynomials.

When the underlying scalar field is complex we show that there are far fewer real extreme points of the unit ball of the space of integral polynomials than in the real case. We are still able to obtain Banach-Stone type theorems and we exhibit a new form of isometric isomorphism which is not seen either in the real or in the homogeneous case. Again, by showing that the centralizer of a certain Banach space is trivial, we are able to extend these results to the vector valued case.

(Anthony Brown) School of Mathematical Sciences, University ColLEge Dublin, Dublin 4

E-mail address, Anthony Brown: anthony.brown@ucd.ie 